\title{
Higher order vortex gyrotropic modes in circular ferromagnetic nanodots
}

SUBJECT AREAS:

MAGNETIC PROPERTIES

AND MATERIALS

FERROMAGNETISM

Received

6 February 2014

Accepted

8 April 2014

Published

25 April 2014

Correspondence and requests for materials should be addressed to A.O.A. (eleaao@nus. edu.sg)

\author{
Junjia Ding', Gleb N. Kakazei', Xinming Liu', Konstantin Y. Guslienko ${ }^{2,3}$ \& Adekunle O. Adeyeye'
}

\begin{abstract}
'Information Storage Materials Laboratory, Department of Electrical and Computer Engineering, National University of Singapore, Singapore-1 17576, Singapore, ${ }^{2}$ Departamento de Física de Materiales, Facultad de Química, Universidad del País Vasco, 20018 San Sebastián, Spain, ${ }^{3}$ KEERASQUE, the Basque Foundation for Science, 48011 Bilbao, Spain.
\end{abstract}

\begin{abstract}
Magnetic vortex that consists of an in-plane curling magnetization configuration and a needle-like core region with out-of-plane magnetization is known to be the ground state of geometrically confined submicron soft magnetic elements. Here magnetodynamics of relatively thick $(50-100 \mathrm{~nm})$ circular $\mathrm{Ni}_{80} \mathrm{Fe}_{20}$ dots were probed by broadband ferromagnetic resonance in the absence of external magnetic field. Spin excitation modes related to the thickness dependent vortex core gyrotropic dynamics were detected experimentally in the gigahertz frequency range. Both analytical theory and micromagnetic simulations revealed that these exchange dominated modes are flexure oscillations of the vortex core string with $n=$ $0,1,2$ nodes along the dot thickness. The intensity of the mode with $n=1$ depends significantly on both dot thickness and diameter and in some cases is higher than the one of the uniform mode with $n=0$. This opens promising perspectives in the area of spin transfer torque oscillators.
\end{abstract}

peritis here is a considerable interest nowadays to topologically non-trivial magnetization states in small magnetic particles and thin films, such as magnetic vortices ${ }^{1-4}$ and skyrmions ${ }^{5,6}$. Vortex state is characterized by inplane curling magnetization configuration and a small $(\sim 10 \mathrm{~nm})$ region of the vortex core with out-of-plane magnetization ${ }^{1}$. Flat magnetic particles (dots) made of soft magnetic material have a vortex state of magnetization as the ground state for certain relations between thicknesses and lateral sizes as a result of competition of the exchange and magnetodipolar energies ${ }^{7}$. The simplest example is a single vortex state with the core located in the center of a circular dot of radius $R$ and thickness $L$ in the absence of external magnetic field. The vortex is considered as one of the most iconic objects of nanomagnetism and often serves as an ideal structure to probe new physical concepts and experimental techniques. Recently a symmetry breaking in the formation process of vortex states was experimentally observed in a single circular ferromagnetic nanodot by magnetic transmission soft Xray microscopy ${ }^{8}$. Other impressive example is a demonstration of scanning nitrogen-vacancy magnetometer capabilities by quantitative measurement of the three-dimensional picture of the stray magnetic field emitted by a vortex in a submicron ferromagnetic square dot $^{9}$.

The experimental study of magnetic vortex dynamics was started in 2003 by using time-resolved Kerr microscopy ${ }^{2}$. Then Novosad et al. ${ }^{10}$ demonstrated that being shifted from its equilibrium position by microwave field, the vortex core experiences sub - GHz oscillations with the eigenfrequency proportional to the thickness/radius aspect ratio $b=L / R$. Later it was shown that similar microwave-frequency oscillations with extremely narrow linewidth can be excited by dc polarized current ${ }^{11,12}$. Finally large microwave generation from current-driven magnetic vortex oscillators was observed in magnetic tunnel junctions ${ }^{13,14}$. These results allow considering the magnetic vortex motion as an important excitation mode of the spin torque oscillators (STO) in layered nanopillars and nanocontacts. Another perspective way is to use a vortex gyration as a propagation mode in dipolarly coupled dot array magnonic crystals ${ }^{15}$.

In all previous experiments the observed vortex gyrotropic mode had a uniform profile along the dot thickness. The non-uniform along thickness $1^{\text {st }}$ order vortex core mode $(n=1)$ was simulated micromagnetically by Boust and Vukadinovic ${ }^{16}$ for small radii circular dots. Then, the existence of higher order gyrotropic modes was demonstrated by micromagnetic simulations for rectangular prisms ${ }^{17}$. These modes can be described as flexure oscillations of the vortex core line with $n=0,1,2$ nodes along the dot thickness. They resemble standing spin-wave resonance modes in perpendicularly magnetized continuous ferromagnetic films ${ }^{18}$. In the present paper the $1^{\text {st }}$ order gyrotropic mode was detected experimentally for the first time using broadband ferromagnetic resonance technique, and the $2^{\text {nd }}$ order mode was observed using micromagnetic simulations. The proposed analytical theory based on Thiele equation ${ }^{19}$ describes the whole vortex gyrotropic spectra in a circular dot accounting exchange and magnetostatic energies. In contrast to uniform gyrotropic mode $(n=0)$ the eigenfrequencies of the higher order ones decrease 
significantly with dot thickness increase and do not depend on dot aspect ratio. The intensity of the mode with $n=1$ depend significantly on both thickness and dot aspect ratio and in some cases became higher than the one of the mode with $n=0$. Finally higher order modes are lying in the gigahertz frequency range. The last two properties makes the mode with $n=1$ extremely attractive for applications in spin-torque oscillators.

\section{Results}

Samples and measurement techniques. Periodic arrays of circular $\mathrm{Ni}_{80} \mathrm{Fe}_{20}$ dots with diameter $d=300 \mathrm{~nm}$ and pitch $p=620 \mathrm{~nm}$ were fabricated on $\mathrm{Si}$ substrate over a large area $(4 \mathrm{~mm} \times 4 \mathrm{~mm})$ using deep ultraviolet lithography at $248 \mathrm{~nm}$ exposure wavelength followed by electron beam evaporation and lift-off process. Details of the processing steps can be found elsewhere ${ }^{20}$. The thickness $L$ of the $\mathrm{Ni}_{80} \mathrm{Fe}_{20}$ dots was varied from $50 \mathrm{~nm}$ to $100 \mathrm{~nm}$. For the $L=$ $60 \mathrm{~nm}$ case, two more samples with $d=430 \mathrm{~nm}$ and $d=550 \mathrm{~nm}$ were prepared additionally during the same processing steps keeping the pitch $p=620 \mathrm{~nm}$. A representative scanning electron microscopy micrograph of the tilted (viewing angle $55^{\circ}$ ) $60 \mathrm{~nm}$ thick $\mathrm{Ni}_{80} \mathrm{Fe}_{20}$ dot array is shown in Fig. 1(a). Despite of the relatively large ratio $b=$ 0.266 , the dots are still looking like flat objects with sharp edges.

The magnetization behavior of the fabricated dot arrays were characterized using focused longitudinal magneto-optical Kerr (MOKE) setup with a spot size of about $5 \mu \mathrm{m}$ in the longitudinal geometry. The representative hysteresis loop of the 60-nm thick $\mathrm{Ni}_{80} \mathrm{Fe}_{20}$ dot array is shown in the Fig. 1(b). The loop has a double triangle shape and zero remanence, typical for the circular dots with the vortex ground state. Vortex nucleation and annihilation fields ${ }^{21}$ are quite pronounced, that is a clear indication of the high quality of the fabrication process.

The microwave absorption spectra of the dot arrays in the symmetric ground state (i.e., in the absence of an external in-plane magnetic field) and at room temperature was measured using a vector network analyzer (VNA: model Agilent E8363C) by sweeping the frequency in the $50 \mathrm{MHz}-6 \mathrm{GHz}$ range with $5 \mathrm{dBm}$ input microwave power and 10-time averaging process. Standard short, open, load, thru (SOLT) calibration was performed before the measurements. To maximize the sensitivity of microwave measurements, a 50 Ohm coplanar waveguide (CPW) with signal line length of $300 \mu \mathrm{m}$, width of $20 \mu \mathrm{m}$ were fabricated on top of the dot arrays. The amplitude of the microwave magnetic field generated in the vicinity of the signal line was calculated from the current in the CPW following the procedure described in Ref. 22 and found to be $\sim 2$ Oe.

Broadband ferromagnetic resonance spectroscopy. The obtained experimental broadband microwave absorption spectra for different dot thicknesses $L$ are presented in Figs. 2 (a)-(d). The frequency range was limited to $6 \mathrm{GHz}$. This allows us to exclude the well studied azimuthal spin wave modes, which for the circular dots of the given sizes are excited at the frequency just above $6 \mathrm{GHz}$. All the spectra contain two well-pronounced resonance peaks with quite different thickness dependence. The lower one is slightly shifting up from $0.8 \mathrm{GHz}$ to $1.0 \mathrm{GHz}$ with initial thickness increase from $L$ $=50$ to $60 \mathrm{~nm}$, and with further thickness increase it returns back to $0.8 \mathrm{GHz}$ for $L=100 \mathrm{~nm}$, i.e. the total frequency shift of the peak is only $0.2 \mathrm{GHz}$. However, the frequency of the higher resonance peak is monotonically decreasing from $5.4 \mathrm{GHz}$ for $L=50 \mathrm{~nm}$ to $2.4 \mathrm{GHz}$ for $100 \mathrm{~nm}$, i.e., by tuning the dot thickness in the 50$100 \mathrm{~nm}$ range, the frequency of the peak is reduced by $3 \mathrm{GHz}$. In addition, the relative intensity of the second peak drastically increases with the dot thickness increasing.

The identification of the experimentally observed peaks was performed using the LLG micromagnetic simulator ${ }^{23}$. For the case when the distance between dot centers is more than twice the dot diameter, interdot dipolar interactions are considered to be negligibly small even in the saturated state ${ }^{24}$. Therefore simulations were carried out for the individual dots. The simulated microwave absorption spectra for the dots with diameter $d=300 \mathrm{~nm}$ and thicknesses varied from 50 to $100 \mathrm{~nm}$ for the frequency range $50 \mathrm{MHz}-6 \mathrm{GHz}$ are presented in Figs. 2. (e) - (h) and demonstrate excellent agreement with the experiment. For simplicity of comparison, intensities of both experimental and simulation spectra were normalized to the intensity of the strongest mode. Both the positions of the two simulated low frequency peaks and their relative intensities are very similar to the experimental ones. Additionally, for $L=80$ and $100 \mathrm{~nm}$ a third peak was observed at higher frequencies. Unfortunately, its intensity is significantly lower than intensity of the two other peaks; therefore it was complicated to be observed in the experiments.

Fig. 2(i) shows the spatial distribution of the magnetization along the excitation field direction of the three observed modes for the dot with $d=300 \mathrm{~nm}$ and $L=100 \mathrm{~nm}$. It is important to note that these modes were excited by the sinusoidal microwave field with amplitude of 2 Oe, i.e., by the same field that was applied in the experiment. All the three modes correspond to the vortex core spin excitations. The lowest one is the uniform vortex gyrotropic mode, since it is excited in the whole thickness range from top to bottom, although its intensity depends a bit on thickness coordinate (no nodes, $\mathrm{n}=0$ ). The second mode has maximum intensity on upper and lower dot surfaces and is not excited in the dot center (one node, $n=1$ ). It is the one that was observed in micromagnetic simulations of Boust and Vukadinovic $^{16}$. Finally, the third mode has the maximum intensity on both the dot faces and in the dot center, with two unexcited areas lying in between (two nodes, $\mathrm{n}=2$ ). Therefore, the last two modes can be considered as the $1^{\text {st }}$ and $2^{\text {nd }}$ resonances of the vortex core line or as high order gyrotropic vortex modes.

Perhaps the most stunning feature of the $1^{\text {st }}$ order vortex gyrotropic mode is the thickness dependence of the mode intensity - it
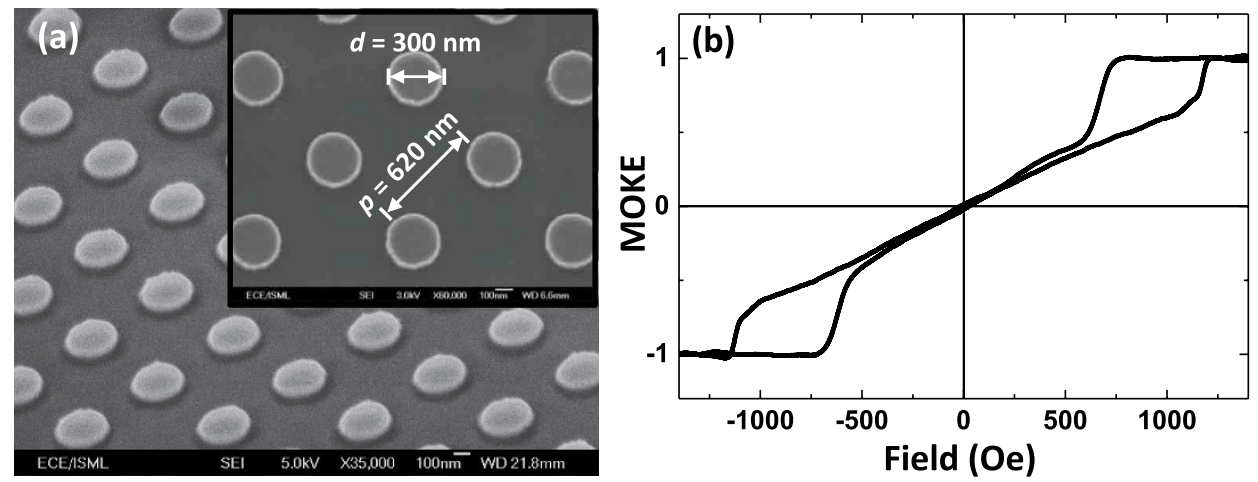

Figure $1 \mid$ (a) Scanning electron microscopy image (the viewing angle is $55^{\circ}$ ) of $\mathrm{Ni}_{80} \mathrm{Fe}_{20}$ dot array. The dot diameter $d=300 \mathrm{~nm}$, pitch $p=620 \mathrm{~nm}$ and thickness $L=60 \mathrm{~nm}$; (b) Hysteresis loop of the dot array presented in Fig. 1(a), measured by focused magneto-optical setup. 

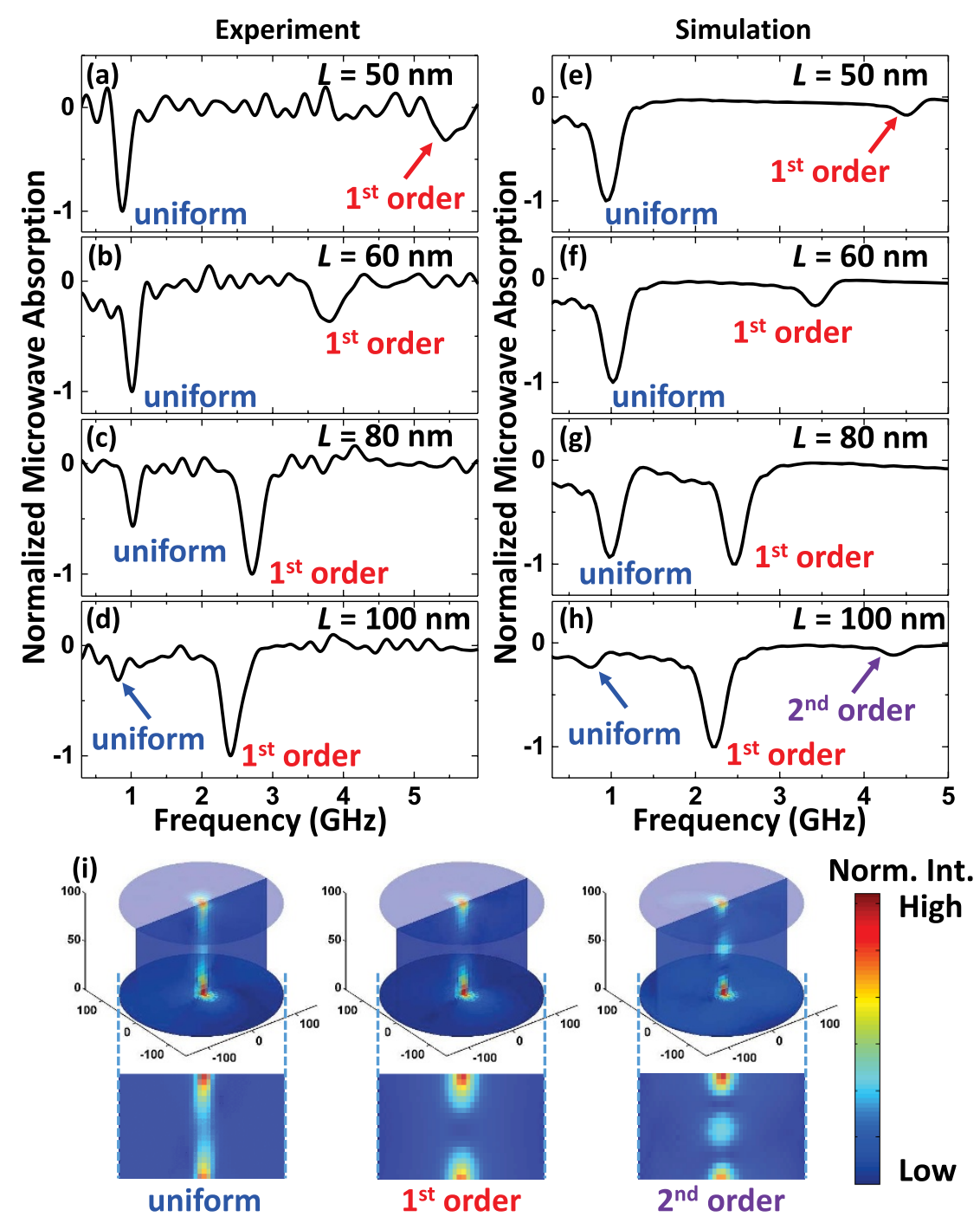

Figure $2 \mid$ (a) - (d) Experimental; and (e) - (h) simulated microwave absorption spectra in the absence of external magnetic field for the dot arrays with diameter $d=300 \mathrm{~nm}$, pitch $p=620 \mathrm{~nm}$, and different values of dot thickness $L$ varied from 50 to $100 \mathrm{~nm}$; (i) Spatial distribution of three lowest eigen-modes for the dot with diameter $d=300 \mathrm{~nm}$ and thickness $L=100 \mathrm{~nm}$.

increases with thickness and becomes more intensive than the uniform gyrotropic mode above $L=80 \mathrm{~nm}$. To clarify this issue, vortex gyration radii $\mathrm{R}_{\mathrm{g}, \mathrm{n}}$ of gyrotropic modes on the dot surface were extracted from micromagnetic simulations by applying microwave field of $2 \mathrm{Oe}$ at the corresponding mode resonance frequencies determined from the spectra excited by the pulse field. It appeared that for $L=60 \mathrm{~nm}$ dot, the gyration radius of uniform mode $\mathrm{R}_{\mathrm{g}, 0} \sim 5 \mathrm{~nm}$ is bigger than the one of $1^{\text {st }}$ mode $\mathrm{R}_{\mathrm{g}, 1} \sim 2 \mathrm{~nm}$, whereas for $100 \mathrm{~nm}$ thick dot the reversed values $-\mathrm{R}_{\mathrm{g}, 0} \sim 3 \mathrm{~nm}$ and $\mathrm{R}_{\mathrm{g}, 1} \sim 6 \mathrm{~nm}$ - were observed. The $2^{\text {nd }}$ mode $\mathrm{R}_{\mathrm{g}, 2}$ has negligibly small value of $\sim 1 \mathrm{~nm}$. There is a clear correlation between mode intensity and gyration radius. High intensity of the $1^{\text {st }}$ gyrotropic mode opens wide perspectives in the area of spin torque oscillators in nanopillars and nanocontacts, where the oscillation power plays a significant role. Of course, to obtain the maximum power at high frequency, thickness and diameter of the nanopillar should be carefully optimized using micromagnetic simulations. It is important to note that typical nanopillar lateral sizes for STO are in the $75-150 \mathrm{~nm}$ range. Our simulations indicate that vortex is still the ground state for the 60 -nm thick disk $(2 R=100 \mathrm{~nm})$ and ellipse (lateral dimensions $75 \times 125 \mathrm{~nm})$ and that the $1^{\text {st }}$ vortex mode for these objects has the higher intensity than the uniform gyrotropic mode.
Analytical theory. To calculate the excitation frequencies of the measured modes we use Landau-Lifshits-Gilbert (LLG) equation of motion, $\dot{\mathbf{M}}=-\gamma \mathbf{M} \times \mathbf{H}+\alpha \mathbf{M} \times \dot{\mathbf{M}} / M_{\mathrm{s}}$, which describes the evolution of magnetization $\mathbf{M}(\mathbf{r}, t)$ in the presence of an effective magnetic field $\mathbf{H}$ and damping. The effective field $\mathbf{H}=-\delta \varepsilon / \delta \mathbf{M}$ is a combination of the external magnetic field, exchange field and the demagnetizing field, and $\varepsilon$ is the total magnetic energy density. For description of the dynamics of magnetic domains the LLG equation can be rewritten in the Thiele form ${ }^{19}$ that allows simplifying the calculations. Thiele's approach is applicable for magnetization distributions that can be characterized by a position of its center $\mathbf{X}(t)$ that can vary with time. To consider thickness dependent vortex excitations we assume that magnetization as a function of the coordinates $\mathbf{r}=(\boldsymbol{\rho}, z)$ and time can be written as $\mathbf{M}(\mathbf{r}, t)=$ $\mathbf{M}(\boldsymbol{\rho}, \mathbf{X}(z, t))$. Then, we can rewrite the dampingless LLG equation as equation for $\mathrm{X}$ :

$$
g_{\alpha \beta} \frac{d X_{\beta}}{d t}=-\frac{\delta E}{\delta X_{\alpha}}
$$

where $E=\int d^{2} \boldsymbol{\rho} \varepsilon$ is the total magnetic energy per unit dot thickness, $\alpha, \beta=x, y$. Introducing the unit vector of magnetization $\mathbf{m}=\mathbf{M} / M_{\mathrm{s}}$, 
the components of the gyrotensor $\widehat{g}$ related to the vortex topological charge can be defined as $g_{\alpha \beta}=\left(M_{s} / \gamma\right) \int d^{2} \boldsymbol{\rho}\left(\partial_{\alpha} \mathbf{m} \times \partial_{\beta} \mathbf{m}\right) \cdot \mathbf{m}, \partial_{\alpha}=$ $\partial / \partial X_{\alpha}$

We apply the Thiele's approach for the magnetic vortex motion in circular dot defining the dimensionless vortex core position as $\mathbf{s}=$ $\mathrm{X} / R\left(s=s_{x}+i s_{y}\right)$. The variational derivative of the magnetic energy of the dot (1) with a shifted magnetic vortex centered in a point $\mathbf{s}\left(s_{x}, s_{y}\right)$ is taken with respect to the vortex core coordinate $\mathbf{s}$. The total magnetic energy $W=L \int d z E$ consists of the magnetostatic and exchange energies and can be calculated as a functional of s. The magnetostatic energy is expressed in the Coulomb type form with the magnetic charges $\rho(\mathbf{r}, \mathbf{s})=-M_{s} \operatorname{div} \mathbf{m}(\mathbf{r}, \mathbf{s})$ as

$$
W_{m}(\mathbf{s})=\frac{1}{2} \int d V d V^{\prime} \frac{\rho(\mathbf{r}, \mathbf{s}) \rho\left(\mathbf{r}^{\prime}, \mathbf{s}\right)}{\left|\mathbf{r}-\mathbf{r}^{\prime}\right|}
$$

and the exchange energy is $W_{e x}(\mathbf{s})=A \int d V \sum_{\alpha}\left(\nabla m_{\alpha}(\mathbf{r}, \mathbf{s})\right)^{2}$ with $A$ being the exchange stiffness. Using $\operatorname{div} \mathbf{m}=2(\mathbf{s} \times \hat{\boldsymbol{\rho}})_{z}$ within two-vortex model (Methods) and integrating over the in-plane coordinates we get the non-local magnetostatic energy and the exchange energy $(z$ is in units of $L$ )

$$
\begin{aligned}
& W_{m}[\mathbf{s}(z)]=\frac{1}{2} V \int d z \int d z^{\prime} \kappa\left(z, z^{\prime}\right) \mathbf{s}(z) \cdot \mathbf{s}\left(z^{\prime}\right), \\
& W_{e x}[\mathbf{s}(z)]=\frac{1}{2} V \int d z\left[\eta\left(\frac{\partial \mathbf{s}}{\partial z}\right)^{2}-\mu \mathbf{s}^{2}(z)\right],
\end{aligned}
$$

where $V=\pi R^{2} L, \kappa\left(z, z^{\prime}\right)$ is defined in Methods, $\mu=2 M_{s}^{2}\left(L_{e} / R\right)^{2}$ is the dot in-plane, and $\eta=M_{s}^{2}\left(L_{e} / L\right)^{2}\left[\ln \left(R / R_{c}\right)+5 / 4\right]$ is the perpendicular to the plane exchange stiffness coefficients calculated within the model, $L_{e}=\sqrt{2 A} / M_{s}$ is the exchange length, the core radius $R_{c}(L) \approx$ $0.68 L_{e}\left(L / L_{e}\right)^{1 / 3}$ is weak function of $L^{10}$.

Solving Eq. (1), see Methods, we find the expression for the eigenfrequencies of the non-uniform along the thickness oscillations of the vortex core (neglecting small $\mu$ )

$$
\frac{\omega_{n}(L)}{\omega_{M}}=\frac{\pi}{8}\left(\frac{L_{e}}{L}\right)^{2}\left[\ln \left(\frac{R}{R_{c}}\right)+\frac{5}{4}\right] n^{2}+2 b \int_{0}^{\infty} d k f_{n}(b k) I^{2}(k),
$$

where $\omega_{M}=\gamma 4 \pi M_{s}$, the functions $f_{n}(x)$ and $I(x)$ are defined in Methods, $b=L / R$ and $n=0,1,2, \ldots$.

The frequency (4) is reduced at $n=0$ to well-known frequency of the uniform gyrotropic mode $\omega_{0}(b)$ calculated in Ref. 25 for the aspect ratio $b \ll 1$. But the frequency $\omega_{0}(b)$ at moderate $b$ should be corrected accounting interaction with the azimuthal spin waves ${ }^{26}$. Corrected expression for the lowest gyrotropic frequency is $\omega_{0}^{c}(b)=\omega_{0}(b)\left[1-3 \omega_{0}(b) / \omega_{M}\right]$. The frequency (4) at $n>0$ decreases almost as $1 / L^{2}$ with the thickness $L$ increasing, whereas the dependence on $R$ is logarithmically weak for submicron dots.

Comparison of experiment, theory and simulations. Fig. 3 represents the comparison of the experiment, LLG micromagnetic simulations and analytical theory for different gyrotropic modes for the dots with diameter $d=300 \mathrm{~nm}$ in the dot thickness range from 50 to $100 \mathrm{~nm}$. In contrast to the uniform mode $(n=0)$, for which the analytical theory predicts accurately the eigenfrequency vs. $L / R$ behavior for small values of $L / R$, but overestimates the frequency values for $L / R>0.1$, for the high order mode with $n=1$ one can find almost perfect quantitative agreement between theory and experiment. It is associated with the fact that the dipole-dominated uniform mode depends on aspect ratio of the dot, whereas the vertical exchange interaction determine the higher order mode

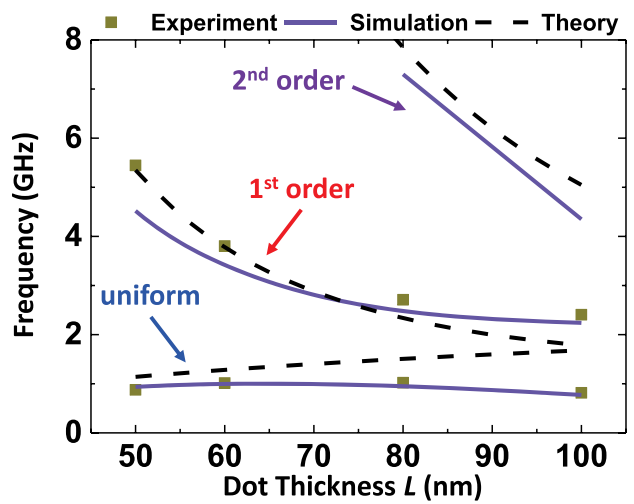

Figure $3 \mid$ Dependence of the uniform $(n=0), 1^{\text {st }}$ and $2^{\text {nd }}$ order gyrotropic mode frequencies $\omega_{n} / 2 \pi$ on the dot thickness $(d=300 \mathrm{~nm})$. Points represent experimental results, solid lines - micromagnetic simulations, dashed lines - analytical calculations by Eq. (4).

frequencies and they depend mainly on the dot thickness. Also a reasonable agreement was observed between theory and micromagnetic simulations for the mode with $n=2$.

To check the last statement, for the dot thickness $L=60 \mathrm{~nm}$, dependence of two lowest resonance peaks $(n=0,1)$ on the dot diameter $d$ was studied. It is necessary to note that for the dots with bigger diameter the dynamic dipolar interactions should be taken into account. However, it was shown in $^{27}$ that for the vortex gyrotropic mode the maximum shift of frequency caused by dipolar interactions is smaller than $50 \mathrm{MHz}$. Experimental results are presented in Fig. 4(a) - (c) and comparison between experiment, theory and simulation is shown in Fig. 4(e). The situation is quite different for the modes with $n=0$ and $n>0$. The frequency of the lowest gyrotropic peak $(n=0)$ is decreasing monotonically from $1 \mathrm{GHz}$ to $0.6 \mathrm{GHz}$ when the dot diameter is increased from 300 to $550 \mathrm{~nm}$ following the $1 / d$ dependence ${ }^{25}$, whereas the frequency of the second peak $(n=1)$ does not change much, i.e. the frequency of the $1^{\text {st }}$ order vortex gyrotropic mode depends only on the dot thickness, but not on the diameter in agreement with Eq. (4).

The remarkable correspondence between measured and simulated microwave absorption spectra for $d=300 \mathrm{~nm}$ dots allow us to use micromagnetic simulations to obtain reliable information regarding properties of higher order gyrotropic modes. Therefore we simulated microwave absorption spectra for dots with $d=100$ and $200 \mathrm{~nm}$ and $L=60 \mathrm{~nm}$. The peak positions are in a good agreement with tendencies presented in Fig. 4, however the intensity of the second $(n=1)$ peak is increasing with diameter decrease and for $d=100 \mathrm{~nm}$ became much bigger than the one of uniform gyrotropic mode (see Fig. 5). Again, this property is extremely promising for the use of vortex mode with $n=1$ in spin-torque nano-oscillators.

\section{Discussion}

Spin excitation modes related to the thickness dependent vortex core gyrotropic dynamics were detected in circular magnetic dots using broadband ferromagnetic resonance and interpreted theoretically. The calculated significant dependence of the high order $(n=$ $1,2, \ldots)$ vortex exchange dominated gyrotropic frequencies on the dot thickness and very weak dependence on the dot diameter was experimentally confirmed for the $1^{\text {st }}$ order mode. This vortex gyrotropic mode of high intensity opens wide perspectives in the area of spin transfer torque oscillators. Despite the fact that most of the modern STO are based on thin magnetic layers (since the spin transfer torque is an interfacial effect), the possibility to excite steady-state oscillation of the main vortex mode at $\sim 1 \mathrm{GHz}$ in $60 \mathrm{~nm}$ - thick Permalloy elliptical dot by dc spin current was demonstrated in Refs. 11,28 . Based on the understanding that the vortex is the ground state 

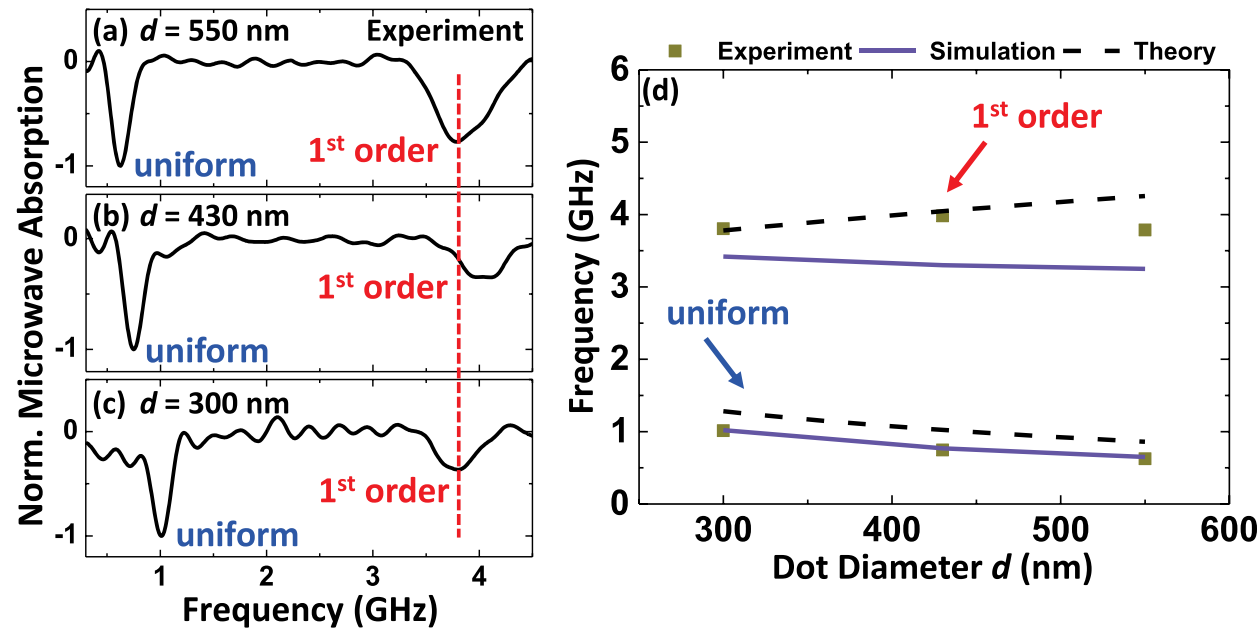

Figure $4 \mid$ (a) - (c) Experimental microwave absorption spectra in the absence of magnetic field for the dot arrays with thickness $L=60 \mathrm{~nm}$, pitch $p=$ $620 \mathrm{~nm}$, and diameter $d$ varied from 300 to $550 \mathrm{~nm}$; (d) Dependence of the uniform and $1^{\text {st }}$ order gyrotropic mode frequencies $\omega_{0,1} / 2 \pi$ on the dot diameter for the dots with $L=60 \mathrm{~nm}$. Points represent experimental results, solid lines - micromagnetic simulations, dashed lines - analytical calculations by Eq. (4).

of magnetic dots of other shapes (square, elliptical, triangular, etc.), we expect that the similar higher-order vortex gyrotropic modes can be observed also in thick nanoelements of different shapes. We consider that there is a close relation between the vortex gyrotropic dynamics and emerging field of magnetic skyrmion dynamics because both the non-linear excitations are kinds of magnetic topological solitons ${ }^{29}$. Therefore, we believe that the vortex gyrotropic modes can shed a light upon essentially more complicated skyrmion dynamics in magnetic films, stripes and dots.

\section{Methods}

Micromagnetic simulation details. Standard parameters for permalloy $\mathrm{Ni}_{80} \mathrm{Fe}_{20}$ (exchange constant $A=1.05 \times 10^{-6} \mathrm{erg} \cdot \mathrm{cm}^{-1}$, gyromagnetic ratio $\gamma=2.93 \mathrm{GHz} /$ kOe and anisotropy constant $K_{U}=0$ ) were used in the simulations. The values of saturation magnetization $M_{s}=810 \mathrm{emu} \cdot \mathrm{cm}^{-3}$ and Gilbert damping parameter $\alpha=$ 0.008 were extracted directly from ferromagnetic resonance frequency vs. resonance field dependence of the reference $60 \mathrm{~nm}$ thick $\mathrm{Ni}_{80} \mathrm{Fe}_{20}$ continuous film. Cell size was fixed at $5 \mathrm{~nm} \times 5 \mathrm{~nm} \times 5 \mathrm{~nm}$. The simulations were carried out for the individual dots, therefore the weak interdot dipolar interactions were not taken into account.

The first step of the dynamic simulations consists of simulating the static magnetization ground states for the dots in the absence of external magnetic field. The ground states obtained are used as initial magnetization states in the dynamic simulations. The dot is then excited by an in-plane pulse field with a full width of $5 \mathrm{~ns}$ and amplitude of 50 Oe. The dot magnetization is left free to evolve following the LLG equation of motion. The spatially averaged magnetization components are then

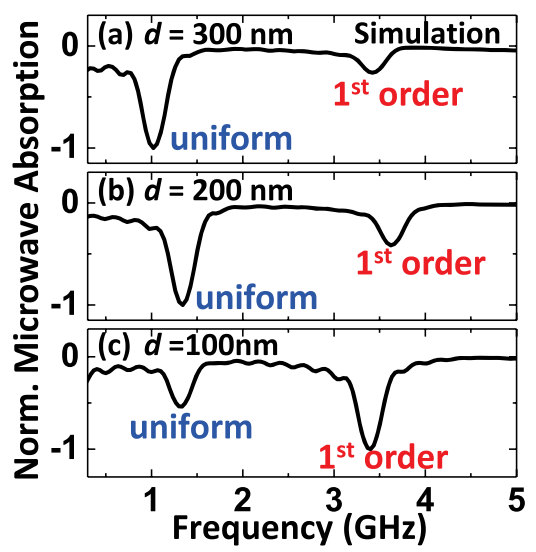

Figure $5 \mid$ (a) - (c) Simulated microwave absorption spectra in the absence of external magnetic field for the dots with thickness $L=60 \mathrm{~nm}$ diameter $d$ varied from 100 to $300 \mathrm{~nm}$. recorded down as a function of time. The dynamic response of the system in frequency domain can be calculated by performing a fast Fourier transform process on the recoded magnetization components. The frequency of the fundamental mode, 1st order and 2nd order mode can be extracted from this simulated dynamic response.

The second step of the dynamic simulation is to generate the magnetization change in time domain by apply a sinusoidal wave excitation field with the corresponding mode frequency (obtained from the first step of simulation). The spatial characteristics of the different modes were quantified by analyzing the results using spatiallyresolved fast Fourier transform imaging ${ }^{30,31}$.

Analytical theory details. The reduced magnetization $\mathbf{m}$ has the following components $m_{x}+i m_{y}=2 w /(1+w \bar{w}), m_{z}=(1-w \bar{w}) /(1+w \bar{w})^{11}$. The complex function $w$ defining mapping of $x y$-plane to the surface of units sphere $|\mathbf{m}|^{2}=1$ is defined as $w=f(\zeta)$ inside the vortex core $|f(\zeta)| \leq 1$, and outside the core, $|f(\zeta)|>1$, $w=f(\zeta) / f(\zeta) \mid$, where $\zeta=(x+i y) / R$. For describing the vortex dynamics we use twovortex model (no side surface magnetic charges) with the analytic function $f(\zeta)$ defined as $f(\zeta)=-(i / c)(\zeta-s)(\bar{s} \zeta-1) /\left(1+|s|^{2}\right), c=R_{d} / R$ is the reduced core radius $^{25}$. It is assumed that the dependence of magnetization on the thickness coordinate $z$ is accounted via the vortex core position $\mathbf{s}(z)$.

The magnetostatic kernel in Eq. (3) is defined as

$\kappa\left(z, z^{\prime}\right)=8 \pi M_{s}^{2} b \int d k e^{-b k\left|z-z^{\prime}\right|} I^{2}(k)$, where $I(k)=\int_{0}^{1} d \rho \rho J_{1}(k \rho)$. Accounting the energies (3) the generalized Thiele equation of motion of the vortex core (1) can be written as

$$
\mathbf{g} \times \dot{\mathbf{s}}=-\frac{\partial w}{\partial \mathbf{s}}+\frac{\partial}{\partial z} \frac{\partial w}{\partial(\partial \mathbf{s} / \partial z)},
$$

where $\mathbf{g}=g_{x y} \hat{\mathbf{z}}, g=2 \pi M_{s} / \gamma$, and $w=w_{m}+w_{e x}, w=E / R^{2}, W=L \int d z E$.

Accounting axial symmetry of the system and assuming a circular motion of the vortex core $\dot{\mathbf{s}}=\boldsymbol{\omega} \times \mathbf{s}$ with the frequency $\omega=|\boldsymbol{\omega}|$, the Thiele equation (M1) can be written as 2-component $1 \mathrm{D}$ Schrödinger equation with a nonlocal potential

$$
\begin{aligned}
& \lambda \mathbf{s}(z)=\left[-\eta \frac{\partial^{2}}{\partial z^{2}}+\hat{U}\right] \mathbf{s}(z), \quad \hat{U} \mathbf{s}(z)=\int d z^{\prime} \kappa\left(z, z^{\prime}\right) \mathbf{s}\left(z^{\prime}\right)-\mu \mathbf{s}(z), \\
& \lambda=g \omega / \pi
\end{aligned}
$$

Eq. (M2) should be amended by the free spin boundary conditions $(\partial \mathrm{s} / \partial z)_{\mathrm{z}}=0, \mathrm{~L}=0$ at the dot faces $z=0, L^{32}$. To solve Eq. (M2) we account that the $\eta$-exchange term dominates for typical dots with $L<R$ (except the case of uniform gyrotropic mode $\mathbf{s}(z)=$ const $)$ and use a perturbation theory considering the $\mathrm{U}$-term as a perturbation. The unperturbed equation $\lambda \mathbf{s}=-\eta \partial^{2} \mathbf{s} / \partial z^{2}$ has the normalized eigenfunctions $u_{n}(z)=\sqrt{2 /\left(1+\delta_{n, 0}\right)} \cos \left(q_{n} z\right)$ and eigenvalues $\lambda_{n}^{0}=\eta q_{n}^{2}$, where quantized wave vector $q_{n}=\pi n(n=0,1,2, \ldots)$ is similar to the case of spin wave resonance in thin magnetic films. The first order perturbation theory yields the eigenvalues

$$
\lambda_{n}=\eta q_{n}^{2}+\langle n|\hat{U}| n\rangle, \quad\langle n|\hat{U}| n\rangle=\int d z d z^{\prime} \kappa\left(z, z^{\prime}\right) u_{n}(z) u_{n}\left(z^{\prime}\right)-\mu
$$


which accounting the relation $\lambda=g \omega / \pi$ determine the discrete vortex eigenfrequencies in Eq. (4) of the main text. Using the eigenfunctions $u_{n}(z)$ the function $f_{n}(x)$ in Eq. (4) is calculated on the basis of Eq. (M3) as

$f_{n}(k)=\frac{k}{k^{2}+q_{n}^{2}}\left(1+\frac{2}{1+\delta_{n, 0}} \frac{k}{k^{2}+q_{n}^{2}}\left[(-1)^{n} e^{-k}-1\right]\right)$.

1. Shinjo, T., Okuno, T., Hassdorf, R., Shigeto, K. \& Ono, T. Magnetic vortex core observation in circular dots of permalloy. Science 289, 930-932 (2000).

2. Park, J. P., Eames, P., Engelbretson, D. M., Berezovsky, J. \& Crowell, P. A. Imaging of spin dynamics in closure domain and vortex structures. Phys. Rev. B 67, 020403 (2003)

3. Jain, S. et al. From chaos to selective ordering of vortex cores in interacting mesomagnets. Nat. Commun. 3, 1330 (2012).

4. Zaspel, C. Gyrotropic frequencies of pinned vortices in magnetic nanodisks. Phys. Rev. B 87, 134425 (2013).

5. Mühlbauer, S. et al. Skyrmion lattice in a chiral magnet. Science 323, 915-919 (2009).

6. Fert, A., Cros, V. \& Sampaio, J. Skyrmions on the track. Nat. Nanotechnol. 8, 152-156 (2013).

7. Cowburn, R. P., Adeyeye, A. O. \& Welland, M. E. Configurational anisotropy in nanomagnets. Phys. Rev. Lett. 81, 5414-5417 (1998).

8. Im, M.-Y. et al. Symmetry breaking in the formation of magnetic vortex states in a permalloy nanodisk. Nat. Commun. 3, 983 (2012).

9. Rondin, L. et al. Stray-field imaging of magnetic vortices with a single diamond spin. Nat. Commun. 4, 2279 (2013).

10. Novosad, V. et al. Magnetic vortex resonance in patterned ferromagnetic dots. Phys. Rev. B 72, 024455 (2005).

11. Pribiag, V. S. et al. Magnetic vortex oscillator driven by d.c. spin-polarized current. Nat. Phys. 3, 498-503 (2007).

12. Mistral, Q. et al. Current-driven vortex oscillations in metallic nanocontacts. Phys. Rev. Lett. 100, 257201 (2008).

13. Dussaux, A. et al. Large microwave generation from current-driven magnetic vortex oscillators in magnetic tunnel junctions. Nat. Commun. 1, 8 (2010).

14. Locatelli, N. et al. Dynamics of two coupled vortices in a spin valve nanopillar excited by spin transfer torque. Appl. Phys. Lett. 98, 062501 (2011).

15. Han, D.-S. et al. Wave modes of collective vortex gyration in dipolar-coupled-dotarray magnonic crystals. Sci. Rep. 3, 2262 (2013).

16. Boust, F. \& Vukadinovic, N. Micromagnetic simulations of vortex-state excitations in soft magnetic nanostructures. Phys. Rev. B 70, 172408 (2004).

17. Yan, M., Hertel, R. \& Schneider, C. M. Calculations of three-dimensional magnetic normal modes in mesoscopic Permalloy prisms with vortex structure. Phys. Rev. B 76, 094407 (2007).

18. Wigen, P. E., Shanabarger, M. R. \& Kooi, C. F. Dynamic pinning in thin-film spinwave resonance, Phys. Rev. Lett. 9, 206-209 (1962).

19. Thiele, A. A. Steady-state motion of magnetic domains. Phys. Rev. Lett. 30, 230 (1973).

20. Adeyeye, A. O. \& Singh, N. Large area patterned magnetic nanostructures. J. Phys. D: Appl. Phys. 41, 153001 (2008).

21. Guslienko, K. Y. Magnetic vortex state stability, reversal and dynamics in restricted geometries. J. Nanosci. Nanotechn. 8, 2745-2760 (2008).

22. Neudecker, I. Magnetization dynamics of confined ferromagnetic systems. Doctoral Dissertation, University of Regensburg (2006) http://epub.uniregensburg.de/10440/1/diss-main.pdf (Date of access: 04.04.2014).
23. Scheinfein, M. R. LLG Micromagnetics Simulator, software for micromagnetic simulations, http://lgmicro.home.mindspring.com (Date of access: 04.04.2014).

24. Kakazei, G. N. et al. Origin of four-fold anisotropy in square lattices of circular ferromagnetic dots. Phys. Rev. B 74, 060406 (2006).

25. Guslienko, K. Y. et al. Eigenfrequencies of vortex state excitations in magnetic submicron-size disks. J. Appl. Phys. 91, 8037-8039 (2002)

26. Guslienko, K. Y., Aranda, G. R. \& Gonzalez, J. Topological gauge field in nanomagnets: Spin-wave excitations over a slowly moving magnetization background. Phys. Rev. B 81, 014414 (2010).

27. Awad, A. A. et al. Spin excitation frequencies in magnetostatically coupled arrays of vortex state circular permalloy dots. Appl. Phys. Lett. 97, 132501 (2010).

28. $\mathrm{Yu}, \mathrm{X}$. W. et al. Images of a spin-torque-driven magnetic nano-oscillator. Phys. Rev. Lett. 106, 167202 (2011).

29. Kosevich, A. M., Ivanov, B. A. \& Kovalev, A. S. Magnetic solitons. Phys. Reports 194, 117-238 (1990).

30. Park, J. P., Eames, P., Engebretson, D. M., Berezovsky, J. \& Crowell, P. A. Spatially resolved dynamics of localized spin-wave modes in ferromagnetic wires. Phys. Rev. Lett. 89, 277201 (2002).

31. Buess, M. et al. Fourier transform imaging of spin vortex eigenmodes. Phys. Rev. Lett. 93, 077207 (2004).

32. Guslienko, K. Y. \& Hoffmann, A. Field evolution of tilted vortex cores in exchange-biased ferromagnetic dots. Phys. Rev. Lett. 97, 107203 (2006).

\section{Acknowledgments}

This work was supported by the SMF-NUS New Horizon Awards, National Research Foundation, Prime Minister's Office, Singapore under its Competitive Research Programme (CRP Award No. NRF-CRP 10-2012-03) and Ministry of Education of Singapore. K.Y.G. acknowledges support by IKERBASQUE (the Basque Science Foundation) and the Spanish MINECO grants PIB2010US-00153, FIS2010-20979-C02-01. The authors would like to thank Dr. N. Singh from A*Star Institute of Microelectronics, Singapore for his help with template fabrication.

\section{Author contributions}

J.D. and X.M.L. fabricated the samples. X.M.L. performed MOKE measurements. J.D. and G.N.K. performed microwave absorption measurements and micromagnetic simulations. K.Y.G. formulated the theoretical problem and performed the analytical calculations. G.N.K. and A.O.A. planned the experiments and simulations. A.O.A. supervised the project. All the authors contributed to the discussion and writing of the manuscript text.

\section{Additional information}

Competing financial interests: The authors declare no competing financial interests.

How to cite this article: Ding, J., Kakazei, G.N., Liu, X.M., Guslienko, K.Y. \& Adeyeye, A.O. Higher order vortex gyrotropic modes in circular ferromagnetic nanodots. Sci. Rep. 4, 4796; DOI:10.1038/srep04796 (2014).

This work is licensed under a Creative Commons Attribution-NonCommercialNoDerivs 3.0 Unported License. The images in this article are included in the article's Creative Commons license, unless indicated otherwise in the image credit; if the image is not included under the Creative Commons license, users will need to obtain permission from the license holder in order to reproduce the image. To view a copy of this license, visit http://creativecommons.org/licenses/by-nc-nd/3.0/ 